\title{
Intervalo entre o Nascimento de Gêmeos: Morbidade e Mortalidade do Segundo Gemelar
}

Time Interval between Twin Delivery: Morbidity and Mortality in the Second Twin

\author{
Jair Luiz Fava ${ }^{1}$, Eduardo de Souza ${ }^{2}$, Luiz Camano ${ }^{2}$
}

\section{RESUM0}

Objetivo: avaliar o efeito do intervalo de tempo entre o nascimento de gêmeos sobre a morbidade e a mortalidade neonatal do segundo gemelar.

Métodos: realizamos um estudo retrospectivo em 50 gemeliparas atendidas na Maternidade Pró-Matre de Vitória que pariram 100 recém-natos vivos, por via vaginal, com peso superior a $500 \mathrm{~g}$ e sem malformações maiores. Separamos os recém-natos em G1 (primeiro gêmeo) e G2 (segundo gêmeo). Foram considerados como indicativos de morbidade neonatal: asfixia ao nascimento, caracterizada pelo indice de Apgar inferior a 7, no $1^{\circ}$ minuto de vida; sindrome do desconforto respiratório, apurada à luz de dados clínicos e radiológicos, e tempo de internação hospitalar superior a 4 dias. Foi analisada também a mortalidade intra-hospitalar. Quanto ao estudo do intervalo de tempo de parturição entre os gêmeos ( $\Delta t)$, realizamos pontos de corte de 5 em 5 minutos, até 35 minutos. Fizemos, também, análise por faixas de tempo: $\Delta t$ até 5 minutos, de 6 a 10, de 11 a 15, de 16 a 20 e de 21 a 150 minutos.

Resultados: não houve diferença estatisticamente significante $(p<0,05)$ entre a morbidade/ mortalidade do segundo gêmeo em relação ao primeiro, considerando os niveis de cortes $e$ faixas de tempo referidos.

Conclusão: o intervalo de tempo de parturição entre G1 e G2 não influenciou a morbidade e mortalidade do segundo gemelar, impondo-se, contudo, na assistência à parturição do segundo gemelar, individualizar as particularidades de cada caso, não contemplando a ansiedade por meio de procedimentos intempestivos e potencialmente danosos.

PALAVRAS-CHAVE: Gemelaridade. Mortalidade perinatal. Anoxia perinatal. Prematuridade.

\section{Introdução}

A motivação de conduzir a parturição gemelar com arte e ciência empolgou inúmeros tocólogos, pois esta representa um dos mais dificeis partos. A assistência às gemeliparas tem representado um grande desafio para os profissionais da Obstetrícia e qualquer plano de conduta requer considerações sobre as várias apresentações, em relação ao primeiro gemelar e segundo

\footnotetext{
${ }^{1}$ Departamentos de Ginecologia e Materno Infantil da Escola de Medicina da Santa Casa de Misericórdia de Vitória Maternidade Pró-Matre de Vitória

${ }^{2}$ Disciplina de Obstetrícia da Universidade Federal de São

Paulo - Escola Paulista de Medicina

Correspondência: Jair Fava

Av. Nossa Senhora da Penha 570, conj. 707-708 - Praia do Canto

29055-912 - Vitória - ES

Fone: (27) 225-2611, Fax: (27) 325-1634

e-mail: jfava@terra.com.br
}

gemelar. A conduta obstétrica nas gemelíparas em que o segundo gemelar exibe apresentações nãocefálicas é especialmente controversa.

$\mathrm{O}$ atendimento à gemelípara deve ser realizado em maternidade de referência e por profissionais com experiência, que devem valorizar alguns dados clínicos, maternos e fetais, denominados de elementos orientadores da conduta na gemelipara ${ }^{1}$. Dentre os elementos de conduta maternos, destacamos a análise da paridade, a presença de cicatriz uterina prévia e as intercorrências clínico-obstétricas. Com relação aos elementos de conduta fetais, acreditamos que os mais importantes são: a apresentação, o peso estimado dos conceptos e a discordância de peso. Esses elementos citados devem ser valorizados pelo tocólogo, não de maneira isolada, mas sim no seu conjunto ${ }^{1}$.

Quanto ao tempo entre o nascimento dos 
gêmeos, velhos textos e aforismas clínicos sugeriam a necessidade do parto do segundo gêmeo ocorrer em um intervalo relativamente pequeno. Ainda em nossos dias, o intervalo de tempo na parturição dos gêmeos e a relação que guarda com a morbidade e mortalidade do segundo gemelar constituem tema controvertido entre os tocólogos ${ }^{2}$. Estudos mais recentes da literatura, de uma forma geral, defendem que o intervalo de nascimento entre os gêmeos não deve ser encurtado às custas de manobras intempestivas. Os recursos da obstetrícia moderna, permitindo melhor acompanhamento da vitalidade fetal, têm favorecido a observação atenta, dependendo das condições obstétricas, sem aumentar a morbidade e a mortalidade neonatal ${ }^{3-10}$. Isto nos motivou a elaborar este estudo objetivando avaliar a morbidade e mortalidade neonatal em gêmeos paridos por via vaginal, em relação ao intervalo de tempo de nascimento entre eles, buscando contribuir para a assistência obstétrica à gemelípara.

\section{Pacientes e Métodos}

Realizamos análise retrospectiva de 50 prontuários de parturientes gemelares, submetidas a parto vaginal, num total de 13.178 partos ocorridos na Maternidade Pró-Matre de Vitória, no período de maio de 1994 a setembro de 1998.

Os RN vivos estudados tinham peso superior a 500 gramas e não apresentavam malformações maiores. Foram alocados em dois grupos, denominados G1 (primeiro gemelar) e G2 (segundo gemelar).

Quanto aos parâmetros adotados para a avaliação da morbidade, seguimos as normas do Serviço de Neonatologia e UTI Neonatal da Maternidade Pró-Matre de Vitória, a seguir enunciados: asfixia perinatal - indicada pelo índice de Apgar no $1^{\circ}$ minuto de vida, avaliado pelos neonatologistas na sala de parto; consideramos como asfixia perinatal valores menores que 7 no $1^{\circ}$ minuto; síndrome do desconforto respiratório (SDR) - caracterizada pela presença de um dos seguintes dados clínicos: cianose, taquidispnéia, esforço respiratório e gemência. Os achados radiológicos dos pulmões (aspecto retículo-granular) foram associados aos achados clínicos, tempo de internação hospitalar (IH) - em consonância com as normas do Serviço de Neonatologia da Pró-Matre de Vitória, que entende como crianças saudáveis as que permanecem internadas por período de até 4 dias; definimos portanto como morbidade presente os recém-nascidos que ultrapassaram este período.
Consideramos óbito neonatal quando o decesso ocorreu durante o período de internação. Separamos os gêmeos (primeiro gemelar e segundo gemelar), quanto à presença de morbidade e mortalidade, em dois grupos: presente e ausente. O grupo presente foi caracterizado quando o gêmeo apresentou uma ou mais morbidades anteriormente definidas e/ou mortalidade. O estudo do intervalo de tempo de parturição entre os gêmeos $(\Delta \mathrm{t})$ objetivou a comparação entre primeiro gemelar e segundo gemelar, quanto aos parâmetros de morbidade e mortalidade. Numa primeira visão, pontos de corte nos Dt de 5 em 5 minutos foram realizados buscando a detecção de dados estatisticamente significantes. A freqüência de casos em cada intervalo $(\Delta \mathrm{t})$ foi: até $5 \mathrm{mi}-$ nutos: 13 gemeliparas; até 10 minutos: 35 gemeliparas; até 15 minutos: 41 gemeliparas; até 20 minutos: 44 gemelíparas; até 25 minutos: 45 gemelíparas e até 35 minutos: 46 gemelíparas.

Outrossim, de outra perspectiva foram analisadas a morbidade e a mortalidade de G2 observando os intervalos de parturição dos gêmeos por faixa de tempo. Assim consideramos os seguintes grupos, conforme o intervalo observado entre os nascimentos: até 5 minutos: 13 pares de gêmeos; de 6 a 10 minutos: 22 pares de gêmeos; de 11 a 15 minutos: 6 pares de gêmeos; de 16 a 20 minutos: 3 pares de gêmeos; de 21 a 150 minutos: 6 pares de gêmeos.

Nossos resultados foram analisados utilizando-se testes não-paramétricos: teste do $\chi^{2}$ para as Tabelas 1 e 2 X N; teste exato de Fisher, quando uma ou mais freqüências esperadas foram menores ou iguais a 5 . Em todos os testes fixamos em 0,05 ou $5 \%$ o nível para rejeição da hipótese de nulidade.

A coleta e publicação dos dados obtidos do prontuáriio foram autorizadas pela Associação Beneficente Pro-Matre de Vitória e o projeto foi aprovado pela Comissão de Ética em Pesquisa do Hospital São Paulo (UNIFESP).

\section{Resultados}

A freqüência de morbidade/mortalidade no segundo gemelar, embora mais alta em todos os intervalos, não foi significantemente diferente daquela observada nos primeiros gemelares, nos intervalos considerados, utilizando-se pontos de corte de 5 em 5 minutos (Tabela 1).

Os resultados também não diferiram estatisticamernte quando o intervalo de parturição dos gêmeos foi avaliado por faixa de tempo (Tabela 
2), embora a morbidade e mortalidade do segundo gemelar tenham sido superiores em todas as fai- xas e mesmo as do primeiro gemelar tenham sido maiores quando o intervalo foi maior.

\begin{tabular}{|c|c|c|c|c|c|c|}
\hline Intervalos & até 5 & até 10 & até 15 & até 20 & até 25 & até 35 \\
\hline $\mathrm{G} 1$ & 23,1 & 45,7 & 46,3 & 45,4 & 44,4 & 45,6 \\
\hline G2 & 30,7 & 51,4 & 53,7 & 52,3 & 53,3 & 52,2 \\
\hline
\end{tabular}

Tabela 2 - Porcentagem de achados (morbidade/mortalidade) do $1^{\circ}$ e $2^{\circ}$ gemelar em relação ao intervalo entre o nascimento, por faixa de tempo em minutos.

\begin{tabular}{cccccc}
\hline Faixa & até & $\mathbf{6}$ a $\mathbf{1 0}$ & $\mathbf{1 1}$ a $\mathbf{1 5}$ & $\mathbf{1 6}$ a 20 & $\mathbf{2 1}$ a 150 \\
\hline $\mathrm{G} 1$ & 23,1 & 59,1 & 50,0 & 33,3 & 50,0 \\
$\mathrm{G} 2$ & 30,7 & 63,6 & 66,7 & 33,3 & 50,0 \\
\hline
\end{tabular}

\section{Discussão}

A polêmica existente em relação ao intervalo de tempo de nascimento entre o primeiro e o segundo gêmeo, paridos pela via vaginal, e suas possiveis repercussões sobre a morbidade e a mortalidade neonatal do segundo gêmeo nos motivou a estudar este tema na Maternidade PróMatre de Vitória. Atualmente, existe escassa publicação sobre este assunto, o que justifica a aplicação de mais pesquisa que vise a novos achados.

Sem dúvida é muito angustiante, para toda a equipe assistencial, o intervalo de tempo que medeia o nascimento de gêmeos na parturição vaginal. A conduta obstétrica moderna obviamente requer assistência mais criteriosa, incluindo condições adequadas de monitorização, com o objetivo de tornar o acompanhamento à gemelípara mais seguro.

Na Disciplina de Obstetrícia da Universidade Federal de São Paulo - Escola Paulista de Medicina, temos preconizado aguardar o parto espontâneo do segundo gemelar cefálico, com rotura das membranas, quando possivel; uso parcimonioso de ocitócicos e monitoração da vitalidade de forma clinica apurada ou com cardiotocografia. Se houver necessidade, praticar o fórcipe respeitando-se as condições de aplicabilidade. Somente em casos excepcionais, na apresentação cefálica de segundo gemelar, seria necessária a versão com extração pélvica, como, por exemplo, no sofrimento fetal agudo por prolapso de cordão ou descolamento prematuro da placenta. Em segundo gemelar pélvico, a norma rotineira é proceder ao parto pélvico com os cuidados habituais. Temos preconizado, ainda, em concordância com outros autores ${ }^{6}$, em segundo gemelar transverso, tentar primeiro a versão externa e, na sua falha, a versão interna ${ }^{1}$. Essas atuações requerem vivência obstétrica por parte do parteiro, cujas decisões devem ser rápidas e precisas, tentando realizá-las dentro de um intervalo de nascimento entre os gêmeos (Dt) razoavelmente curto. Esses aspectos assistenciais à gemelípara nos estimularam a pesquisar o tempo de nascimento de segundo gemelar em relação ao primeiro gemelar, visto que essas manobras geralmente requerem cautela, delicadeza e razoável tempo na sua execução.

Nossa população foi composta por 50 gemeliparas, constituindo, a nosso ver, uma casuística suficiente para uma primeira avaliação, em que estudamos o intervalo entre os nascimentos em relação à morbidade e/ou mortalidade presentes no primeiro e no segundo gêmeo (G1 e G2) paridos por via vaginal.

Estamos cientes das críticas atribuiveis a análises retrospectivas como a nossa. Entretanto, a grande maioria dos relatos da literatura é composta de estudos desse gênero, visto que a realização de avaliação prospectiva levaria muitos anos para ser concluída.

Optamos pela análise conjunta das morbidades e mortalidade devido ao tamanho de nossa população, objetivando uma primeira visão global do valor do intervalo entre os pontos em nossa maternidade. Provavelmente, entre as morbidades consideradas, a mais polêmica relaciona-se ao índice de Apgar como forma de caracterizar a asfixia perinatal. Gostariamos de salientar que a Maternidade Pró-Matre de Vitória não dispõe de alguns recursos que poderiam melhor avaliar o desfecho neonatal, como a dosagem do pH no sangue do cordão umbilical; entretanto, alguns autores também utilizaram o índice de Apgar 
em estudos similares ao nosso ${ }^{2,5,11}$. Por outro lado, o escore de Apgar continua a ser usado na grande maioria dos hospitais brasileiros, principalmente devido à sua simplicidade e praticidade, constituindo método útil auxiliar na orientação das manobras de reanimação empregadas.

Ao iniciarmos nosso estudo, esperávamos que ocorresse uma elevação na morbidade e mortalidade do segundo gemelar, decorrente do aumento do intervalo de tempo de nascimento do mesmo. Ao vermos que o desfecho neonatal não sofreu modificações com a primeira análise estatística, realizada por meio da observação do intervalo em cortes de 5 em 5 minutos (até 35 minutos), procuramos analisar os dados de forma mais detalhada promovendo uma nova visão dos números, separando-os por faixas de tempo. Percebemos que, efetivamente, a extensão do intervalo em nosso trabalho, nessa população analisada da forma proposta, não interferiu com a morbidade e mortalidade do segundo gemelar parido por via vaginal. Não estamos sozinhos nessa verificação. Vários autores chegaram a conclusões semelhantes, mostrando que este parâmetro tem um valor relativo e não absoluto no desfecho neonatal do segundo gemelar-8, 12,13.

É nosso objetivo futuro a realização de estudo mais amplo, com casuística maior, buscando analisar as morbidades separadamente, como o fizeram alguns investigadores.

Evidentemente sabemos que o desfecho neonatal no segundo gemelar sofre influência direta da apresentação, paridade, número de câmaras amnióticas, anestesia, local do parto, experiência do obstetra, existência de ultra-sonografia na sala de parto, assim como da cardiotocografia, existência de alguma patologia clinica e/ou obstétrica associada, entre outros fatores. Devido a estes fatos, fizemos questão de comparar o segundo gemelar com o primeiro gemelar, seu irmão, como forma de diminuir a influência de alguns destes fatores.

Outro aspecto importante de nossa metodologia que merece ser destacado é que não estudamos separadamente a nossa população de RN no que diz respeito ao peso ou à idade gestacional. É evidente que sabemos da importância desta separação. Camano et al. ${ }^{14}$ atribuíram o prognóstico destes conceptos diretamente à prematuridade. É nosso desejo, num próximo estudo, nos ocupar de promover análise mais pormenorizada de nosso material com casuística maior.

Não temos a pretensão de afastar o valor do intervalo como fator de risco para complicações. Não o documentamos em nossa análise, mas isso não quer dizer que defendemos uma atitude exclusivamente expectante diante do parto do segun- do gemelar. Julgamos importante salientar que os nossos dados falam a favor de evitarmos atitudes intempestivas, potencialmente traumáticas e afoitas, em relação ao parto do segundo gemelar.

Surpreendentemente, a mortalidade neonatal intra-hospitalar em nosso estudo não foi expressiva. Este fato talvez reflita uma melhor assistência neonatal, mas não garante ausência de seqüelas além do período neonatal, fato que temos como desejo identificar em um próximo estudo.

Os casos devem ser individualizados, mas provavelmente o prognóstico não será afetado se houver a demora de alguns minutos na avaliação do feto, na chegada de um obstetra mais experiente, ou para ministração de anestesia. De acordo com os dados obtidos em nosso trabalho, a tentativa de versão externa é pertinente, uma vez que poucos minutos são gastos com esse tipo de manobra, não havendo necessidade de precipitação na conduta obstétrica.

Entendemos que estas nossas primeiras avaliações são importantes e julgamos fundamental prosseguir a pesquisa sobre a influência do intervalo de tempo de nascimento entre gêmeos, ampliando a casuística e analisando separadamente as variáveis, para que possamos trazer maior contribuição à condução do parto do segundo gêmeo.

Concluímos que, apesar de não apurarmos alteração relevante da morbidade e mortalidade do segundo recém-nato, parido por via vaginal, em relação ao intervalo de tempo de nascimento entre os gêmeos, impõe-se, na assistência à parturição do segundo gemelar, individualizar as particularidades de cada caso, recomendando-se não contemplar a ansiedade por meio de procedimentos intempestivos.

\section{SUMMARY}

Purpose: to evaluate the impact of birth time interval between twins in relation to the morbidity and mortality of the second newborn twin.

Methods: this is a retrospective study of 50 women with twin pregnancy at the 'Maternidade Pró-Matre de Vitória' who gave birth to 100 alive newborns through the vaginal canal, weighing each over $500 \mathrm{~g}$ and not having any major malformations. The newborns were separated into two groups: G1 (first twin) and G2 (second twin). The following criteria were considered to be indicative of newborn morbidity: asphyxia at birth, characterized by Apgar index lower than 7 in the first minute of life; respiratory distress syndrome detected by clinical and radiological findings, and hospitalization period longer than 4 days. In-hospital 
mortality was analyzed as well. Cut off was at every 5 minutes, up to 35 minutes, to study the time interval between the twin $(\Delta t)$ delivery. A time-span ( $\Delta t)$ analysis was also carried out up to 5 minutes: 6-10, 11-15, 16-20, and 21-150 minutes.

Results: there were no statistically significant differences between the second twin's morbidity/mortality in relation to the first, considering the aforesaid cutoff and time-span levels. Conclusion: delivery time interval between G1 and G2 did not influence the second twin's morbidity and mortality; however, in the assistance of the second twin's delivery, it is absolutely necessary to individualize the characteristics of each case, not allowing any anxiety to set in through untimely and potentially damaging procedures.

KEY WORDS: Twins. Perinatal mortality. Fetal distress. Prematurity.

\section{Referências}

1. Camano L, Guazzelli CAF, Matsubara RL, Bertini AM. Gestação gemelar. In: Prado FC, Ramos JA, Valle JR, editores. Atualização Terapêutica. 18a.ed. São Paulo: Artes Médicas; 1999. p.927-9.

2.Schroder W. Risk assessment of active obstetrical intervention in relation to delivery time interval in the control of vaginal twin delivery. Geburtshilfe Frauenheilkd 1990; 50:447-53.

3.Rydhstrom $\mathrm{H}$, Ingemarsson I. Interval between birth of the first and second twin and its impact on second twin perinatal mortality. J Perinat Med 1990; 18:449-53.

4.Bartnicki J, Meyenburg M, Saling E. Time interval in twin delivery: the second twin need not always be born shortly after the first. Gynecol Obstet Invest 1992; 33:19-20.
5.Rayburn WF, Lavin JP, Miodovnik M, Varner MW. Multiple gestation: time interval between delivery of the first and the second twins. Obstet Gynecol $1984 ; 63: 502-6$.

6.Chervenak FA, Johnson RE, Berkowitz RL, Grannum $\mathrm{P}$, Hobbins JC. Is routine cesarean section necessary for vertex-breech and vertextransverse twin gestations? Am J Obstet Gynecol 1984; 148:1-5.

7.Chervenak FA, Johnson RE, Youcha S, Hobbins JC, Berkowitz RL. Intrapartum management of twin gestation. Obstet Gynecol 1985; 65:119-24.

8.Chitkara U, Berkowitz RL. Multiple pregnancy. In: Gabbe SG, Niebyl JR, Simpson JL, editors. Obstetrics: normal and problem pregnancies. $1^{\text {st }}$ ed. Edinburgh: Churchill Livingstone; 1991. p.881-921.

9.Eskes TK, Timmer H, Kollée LA, Jongsma HW. The second twin. Eur J Obstet Gynecol Reprod Biol 1985; 19:159-66.

10.Silva JCG, Milanez HM. Gestação múltipla. In: Neme B, editor. Obstetrícia Básica. 1a ed. São Paulo: Sarvier; 1995. p.185-210.

11.Blickstein I, Shoham-Schwartz Z, Lancet M, Borenstein R. Characterization of the growthdiscordant twin. Obstet Gynecol 1987; 70:11-5.

12.Little WA, Friedman EA. The twin delivery: factors influencing second twin mortality. Obstet Gynecol Surv 1958; 13:611-23.

13.Thompson JP, Johnson CE. Survival and management of the second-born twin. Obstet Gynecol 1966; 27:827-32.

14.Camano L, Delascio D, Amed AM, Sismotto O, Arie MHA. A prematuridade e o baixo peso no gemelar. Rev Bras Ginecol Obstet 1981; 3:47-51.

\section{FAÇA UMA VISITA \\ A HOME PAGE \\ DA FEBRASGO}

www.febrasgo.org.br 